\title{
Outage Performance of Opportunistic Amplify-and-Forward Relaying over Asymmetric Fading Environments
}

\author{
Sudhan Majhi ${ }^{1,2,3}$, Youssef Nasser ${ }^{1,2,3}$, Jean François Hélard ${ }^{1,2,3}$ \\ ${ }^{1}$ European University of Brettany, Rennes, France \\ ${ }^{2}$ Institut National des Sciences Appliquees, Rennes, France \\ ${ }^{3}$ Institute of Electronics and Telecommunications of Rennes, Rennes, France \\ E-mail: \{sudhan.majhi,youssef.nasser, jean-francois.helard\}@insa-rennes.fr \\ Received February 16, 2010; revised March 21, 2010; accepted April 22, 2010
}

\begin{abstract}
This letter analyzes the outage probability of opportunistic amplify-and-forward relaying over asymmetric and independent but non-identically distributed (i.n.d) fading environments. The work investigates the scenarios where cooperative nodes are located at different geographical locations. As a result, the different signals are affected by different i.n.d fading channels, one may undergo Rician fading distribution and others may undergo Rayleigh fading distribution. In this letter, a lower bound of the outage probability for various asymmetric fading environments is derived at high SNR by applying the initial value theorem. The analytical model is validated through Monte-Carlo simulation results.
\end{abstract}

Keywords: Outage Probability, Opportunistic Relaying, Amplify-and-Forward Relaying, Rayleigh and Rician Fading Channels, Asymmetric Fading Channels, Independent and Non-Identically Distributed

\section{Introduction}

Cooperative relaying is a promising technology for future wireless communications. It can benefit most of the leverages of multiple input multiple output (MIMO) without using the conventional MIMO schemes [1]. Among the cooperative techniques, the opportunistic relaying, in which only one relay $(\mathrm{R})$ node forwards the source's $(\mathrm{S})$ data to the destination (D) has shown its efficiency compared to other techniques [2].

The outage performance of opportunistic amplify-andforward (AF) relaying over a symmetric fading environment is widely investigated in $[1,3,4]$. However, in practice, cooperative nodes are usually located in different geographical location environments and at different distances with respect to $\mathrm{S}$ and $\mathrm{D}$. Therefore, one link could be either in line-of-sight (LOS) situation or in non-LOS (NLOS) situation. For example, the fixed relay nodes used for forwarding source's data to a specific region (e.g. tunnel, behind the building) often use directional antenna, so the R-D link is usually in a LOS situation. However, we cannot assume such a situation in all transmission environments especially when $\mathrm{D}$ is in a deep shadowing region with respect to $\mathrm{S}$. The outage performance analysis of opportunistic relaying for mixed and i.n.d fading environments is, therefore, of practical importance.

The asymmetric fading channel is introduced in [5]. However, the authors of this work assume additive white Gaussian noise (AWGN) channel of the R-D link. In [6], an approximation of the outage performance over asymmetric fading channel, i.e., Rayleigh and Rician, is given. However, to the best knowledge of the authors, no closed-form expression is provided. In this letter, the analytical model of the outage probability of opportunistic AF relaying over asymmetric and i.n.d fading environments is given. Then, the lower bound of the outage probability for high SNR values is deduced and verified through Monte-Carlo simulations.

\section{System Model and SNR Evaluation}

In this framework, we consider a general 2-hop AF relaying network consisting of $\mathrm{S}, m$ relays, $\mathrm{R}_{i}, i=1,2, \ldots, m$ and $\mathrm{D}$. We assume that $\mathrm{D}$ performs maximum ratio combining at the receiving side. The equivalent instantaneous end-to-end signal-to-noise ratio (SNR) for opportunistic 
AF relaying is given as [3]:

$$
S N R_{d}=\frac{P_{s}\left|h_{s d}\right|^{2}}{N_{s d}}+\max _{i} \frac{\frac{P_{s}\left|h_{s r_{i}}\right|^{2}}{N_{s r_{i}}} \frac{P_{s}\left|h_{r_{i} d}\right|^{2}}{N_{r_{i} d}}}{\frac{P_{s}\left|h_{s r_{i}}\right|^{2}}{N_{s r_{i}}}+\frac{P_{s}\left|h_{r_{i}}\right|^{2}}{N_{r_{i} d}}+1}
$$

where $\left|h_{a b}\right|^{2}$ represents the channel gain of the a-b link, $P_{a}$ is the power transmitted by the node a. As mentioned in [3], we assume that AWGN variance is $N_{a b}=1 / \gamma_{0}$, $\forall a, b$ where $\gamma_{0}$ is proportional to the system SNR.

For simplicity reasons, we use different notations of the random variables of the different fading distributions. For the Rayleigh fading, let $\gamma_{a b}=P_{a}\left|h_{a b}\right|^{2}$ be the instanttaneous signal power and for the Rician fading, the instantaneous signal power is denoted as $\xi_{a b}$. The probability density function (PDF) of $\gamma_{a b}$ and $\xi_{a b}$ are expressed respectively as:

$$
\begin{gathered}
f_{\gamma_{a b}}(x)=\frac{1}{\bar{\gamma}_{a b}} e^{-x / \bar{\gamma}_{a b}} \\
f_{\xi_{a b}}(\xi)=\frac{K_{a b}+1}{\bar{\xi}_{a b}} e^{-\xi\left(K_{a b}+1\right) / \bar{\xi}_{a b}-K_{a b}} I_{0}\left(\sqrt{\frac{4 K_{a b}\left(K_{a b}+1\right) \xi}{\bar{\xi}_{a b}}}\right)
\end{gathered}
$$

where, $\bar{\gamma}_{a b}=E\left\{\gamma_{a b}\right\} \quad \bar{\xi}_{a b}=E\left\{\xi_{a b}\right\} \quad$ and $\quad K_{a b}$ is the Rician factor. $E\{$.$\} holds for expectation value.$

The upper bound of the instantaneous SNR of (1) for the opportunistic AF relaying is defined as:

$$
S N R_{u b}=P_{s}\left|h_{s d}\right|^{2} \gamma_{0}+\max _{i}\left(\min \left(P_{s}\left|h_{s r_{i}}\right|^{2}, P_{s}\left|h_{r_{i}}\right|^{2}\right)\right)
$$

This instantaneous SNR value will be used in the following section to evaluate the outage probability.

\section{Analysis of Outage Probability}

In this section, we provide the lower bound of the outage probability of opportunistic AF relaying for different channels given in Figure 1.

\subsection{Asymmetric Channel I}

Theorem 1: If S-D link is Rayleigh fading channel and S-R and R-D links are Rician fading channels, then the lower bound of the outage probability over asymmetric channel I is:

$$
p_{\text {out }}^{I}=\frac{1}{(m+1) \bar{\gamma}_{s d}} \prod_{i=1}^{m}\left(\frac{\left(K_{s r_{i}}+1\right)}{\bar{\xi}_{s_{i}} e^{K_{s_{i}}}}+\frac{\left(K_{r_{i} d}+1\right)}{\bar{\xi}_{i_{i}} e^{K_{r_{i} d}}}\right) \gamma^{m+1}
$$

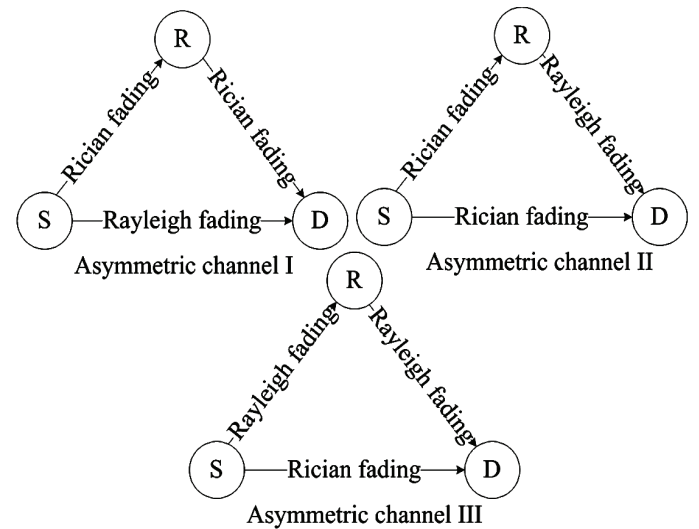

Figure 1. Different asymmetric fading channels of a cooperative network.

Proof: By using $\gamma_{s d}=P_{s}\left|h_{s d}\right|^{2}, \quad \xi_{s r_{i}}=P_{s}\left|h_{s r_{i}}\right|^{2}$ and $\xi_{r_{i} d}=P_{s}\left|h_{r_{i} d}\right|^{2}$ in [4], the outage probability over the asymmetric channel I can be written as:

$$
p_{\text {out }}^{I}=\operatorname{Pr}\left[\gamma_{u b}^{I}<\gamma\right]
$$

where $S N R_{u b}$ is derived to $\gamma_{u b}^{I}=\gamma_{s d}+\xi_{\max }, \quad \gamma=$ $\left(2^{2 R}-1\right) / \gamma_{0}, \quad \xi_{\max }=\max \left(\xi_{1}, \xi_{2}, \ldots, \xi_{m}\right)$ and $\xi_{i}=$

The cumulative distribution function (CDF) of the random variable $\xi_{i}$ over i.n.d is given as:

$$
\begin{aligned}
F_{\xi_{i}}(\gamma)=1 & -\left(1-\operatorname{Pr}\left[\xi_{s_{i}}<\gamma\right]\right)\left(1-\operatorname{Pr}\left[\xi_{r_{i} d}<\gamma\right]\right) \\
= & 1-Q_{1}\left(\sqrt{2 K_{s r_{i}}}, \sqrt{\frac{2\left(K_{s r_{i}}+1\right) \gamma}{\xi_{s r_{i}}}}\right) \times \\
& Q_{1}\left(\sqrt{2 K_{r_{i} d}}, \sqrt{\frac{2\left(K_{r_{i} d}+1\right) \gamma}{\xi_{r_{i} d}}}\right)
\end{aligned}
$$

where $Q_{1}($,$) is the 1^{\text {st }}$ order Marcum Q-function and the PDF of $\xi_{i}$ is obtained by differentiating above as:

$$
\begin{aligned}
f_{\xi_{i}}(\gamma)= & \left(\sqrt{2 K_{s r_{i}}}, \sqrt{\left.\frac{2\left(K_{s r_{i}}+1\right) \gamma}{\bar{\xi}_{s r_{i}}}\right) f_{\xi_{r_{i}}}(\gamma)+}\right. \\
& Q_{1}\left(\sqrt{2 K_{r_{i} d}}, \sqrt{\frac{2\left(K_{r_{i} d}+1\right) \gamma}{\bar{\xi}_{r_{i} d}}}\right) f_{\xi_{s_{i}}}(\gamma)
\end{aligned}
$$

The CDF of the random variable $\xi_{\max }$ over i.n.d fading channel can be expressed as:

$$
F_{\xi_{\max }}(\gamma)=\prod_{i=1}^{m} F_{\xi_{i}}(\gamma)
$$

and the corresponding PDF of $\xi_{\max }$ is obtained by differentiating the above as: 


$$
f_{\xi_{\max }}(\gamma)=\sum_{i=1}^{m} f_{\xi_{i}}(\gamma) \prod_{\substack{j=1 \\ j \neq i}}^{m} F_{\xi_{j}}(\gamma)
$$

Since $F_{\xi_{0}}(0)=0$, the $(m-1)$ th order derivative of (10) at high SNR i.e., at $\gamma=0$ as $\gamma_{0} \rightarrow \infty$, can be written as:

$$
\left.\frac{\partial^{m-1}}{\partial \gamma^{m-1}} f_{\xi_{\max }}(\gamma)\right|_{\gamma=0}=m ! \prod_{i=1}^{m} f_{\xi_{i}}(0)
$$

The outage probability given in (6) is a CDF of $\gamma_{u b}^{I}$, which can be evaluated by using the initial value theorem (IVT) of the Laplace Transformation (LT). The LT of the PDF of the random variable $\gamma_{u b}^{I}$ can be expressed by using Equation 15 in [3] and, then (11), as:

$$
\begin{aligned}
L\left\{f_{\gamma_{u b}}^{I}(\gamma)\right\}= & \left.\frac{1}{s^{m+1}} f_{\gamma_{s d}}(0) \frac{\partial^{m-1}}{\partial \gamma^{m-1}} f_{\xi_{\max }}(\gamma)\right|_{y=0} \\
& =\frac{m !}{s^{m+1}} f_{\gamma_{s d}}(0) \prod_{i=1}^{m} f_{\xi_{i}}(0)
\end{aligned}
$$

Since $f_{\gamma_{d}}(0)$ and $f_{\xi_{i}}(0)$ are constant with respect to the variable $S$, the PDF of $\gamma_{u b}^{I}$ is obtained by applying the inverse LT (ILT) on (12) as:

$$
f_{\gamma_{u b}}^{I}(\gamma)=\gamma^{m} f_{\gamma_{s d}}(0) \prod_{i=1}^{m} f_{\xi_{i}}(0)
$$

We complete the proof by integrating (13) and substituting the vale of $f_{\gamma_{s d}}(0)$ and $f_{\xi_{i}}(0)$.

\subsection{Asymmetric Channel II}

Theorem 2: If S-D and S-R links are Rician fading channels and R-D link is Rayleigh fading channel, then the lower bound of the outage probability over asymmetric channel II is:

$$
p_{\text {out }}^{I I}=\frac{\left(K_{s d}+1\right)}{(m+1) \bar{\xi}_{s d} e^{K_{s d}}} \prod_{i=1}^{m}\left(\frac{\left(K_{s_{i}}+1\right)}{\bar{\xi}_{s r_{i}} e^{K_{s r_{i}}}}+\frac{1}{\bar{\gamma}_{r_{i} d}}\right) \gamma^{m+1}
$$

Proof: For the asymmetric channel II, we use $\xi_{s d}=P_{s}\left|h_{s d}\right|^{2}, \quad \xi_{s r_{i}}=P_{s}\left|h_{s r_{i}}\right|^{2}$ and $\gamma_{r_{i} d}=P_{s}\left|h_{r_{i} d}\right|^{2}$. The outage probability can be expressed as:

$$
p_{\text {out }}^{I I}=\operatorname{Pr}\left[\gamma_{u b}^{I I}<\gamma\right]
$$

where $\gamma_{u b}^{I I}=\xi_{s d}+g_{\max }, \quad g_{\max }=\max \left(g_{1}, g_{2}, \ldots, g_{m}\right)$ and $g_{i}=\min \left(\xi_{s r_{i}}, \gamma_{r_{i} d}\right)$. The CDF of the random variable $g_{i}$ over i.n.d fading channel can be written as:

$$
F_{g_{i}}(\gamma)=1-Q_{1}\left(\sqrt{2 K_{s r_{i}}}, \sqrt{\frac{2\left(K_{s r_{i}}+1\right) \gamma}{\bar{\xi}_{s r_{i}}}}\right) \times\left(1-F_{\gamma_{r^{d}}}(\gamma)\right)
$$

where $F_{\gamma_{r d}}(\gamma)$ is the CDF of the random variable $\gamma_{r_{i} d}$. The corresponding PDF of $g_{i}$ is expressed as:

$$
\begin{aligned}
f_{g_{i}}(\gamma)= & Q_{1}\left(\sqrt{2 K_{s r_{i}}}, \sqrt{\frac{2\left(K_{s r_{i}}+1\right) \gamma}{\bar{\xi}_{s r_{i}}}}\right) f_{\gamma_{r d}}(\gamma)+ \\
& \left(1-F_{\gamma_{r_{d} d}}(\gamma)\right) f_{\xi_{s_{i}}}(\gamma)
\end{aligned}
$$

Similarly, by using the IVT and the ILT, the PDF of $\gamma_{u b}^{I I}$ can be derived as:

$$
f_{\gamma_{u b}}^{I I}(\gamma)=\gamma^{m} f_{\xi_{s d}}(0) \prod_{i=1}^{m} f_{g_{i}}(0)
$$

By integrating (18), we complete the proof.

\subsection{Asymmetric Channel III}

Theorem 3: If S-D link is Rician fading channel and S-R and R-D link are Rayleigh fading channels, the corresponding lower bound of outage probability is:

$$
p_{\text {out }}^{\text {III }}=\frac{\left(K_{s d}+1\right)}{(m+1) \bar{\xi}_{s d} e^{K_{s d}}} \prod_{i=1}^{m}\left(\frac{1}{\bar{\gamma}_{s_{i}}}+\frac{1}{\bar{\gamma}_{r_{i} d}}\right) \gamma^{m+1}
$$

Proof: For the asymmetric channel III, we use $\xi_{s d}=P_{s}\left|h_{s d}\right|^{2}, \gamma_{s r_{i}}=P_{s}\left|h_{s r_{i}}\right|^{2}$ and $\gamma_{r_{i} d}=P_{s}\left|h_{r_{i} d}\right|^{2}$. The outage probability can be written as:

$$
p_{\text {out }}^{I I I}=\operatorname{Pr}\left[\gamma_{u b}^{I I I}<\gamma\right]
$$

where $\gamma_{u b}^{I I I}=\xi_{s d}+\gamma_{\max }, \quad \gamma_{\max }=\max \left(\gamma_{1}, \gamma_{2}, \ldots, \gamma_{m}\right)$ and $\gamma_{i}=\min \left(\gamma_{s_{i}}, \gamma_{r_{i} d}\right)$. The corresponding PDF of the random variable $\gamma_{i}$ is given by:

$$
f_{\gamma_{i}}(\gamma)=\left(1-F_{\gamma_{s_{i}}}(\gamma)\right) f_{\gamma_{\eta^{d}}}(\gamma)+\left(1-F_{\gamma_{\eta^{d}}}(\gamma)\right) f_{\gamma_{s_{i}}}(\gamma)
$$

Again by using the IVT and ILT, the PDF of $\gamma_{u b}^{I I I}$ is obtained as:

$$
f_{\gamma_{u b}}^{I I I}(\gamma)=\gamma^{m} f_{\xi_{s d}}(0) \prod_{i=1}^{m} f_{\gamma_{i}}(0)
$$

By integrating (22), we complete the proof.

Similarly, the outage probability of other possible asymmetric channels can be derived by using the above procedure. The upper bound of the outage probability of the opportunistic AF relaying can be derived simply by using the above method and Equation 8 in [7].

\section{Numerical Examples}

In this section, analytical and Monte-Carlo simulation results are presented. Since the channels are i.n.d, we set different means for different S-Ri/Ri-D links. In the 
Rician fading channel, the Rician factor $K_{a b}$ is uniformly distributed in [2,3] and the mean $\bar{\gamma}_{a b}$ of the NLOS components are uniformly distributed in $[0,1]$. The LOS components are derived for a given $K_{a b}$ and $\bar{\gamma}_{a b}$.

It is clear from (5), (14) and (19) that the outage probability over Rician fading channel is obtained by substituting $1 / \bar{\gamma}_{s d}=\left(K_{s d}+1\right) e^{K_{s d}} / \bar{\xi}_{s d}$ in (5) and the outage probability over Rayleigh fading channel is obtained by substituting $K_{s d}=0$ in (19). Figure 2 shows the lower bound of the outage probability over the symmetric and asymmetric fading environments. Due to the presence of LOS signal, the outage performance over Rician fading channel outperforms all other scenarios. Inversely, due to the absence of direct signal, the Rayleigh fading channel has poorer outage performance than the other scenarios.

The opportunistic relaying provides better outage performance than without cooperation. It implies that the outage performance of opportunistic relaying depends mainly on cooperative links (S-R and R-D links). For this reason, asymmetric channel I provides better outage performance than the asymmetric channel II and asymmetric channel III due to the presence of LOS signal inboth S-R and R-D links.

We also note that asymmetric channel II provides better outage performance than asymmetric channel III. Since S-D and R-D links undergo the same fading in both scenarios, the LOS component existing in S-R link of

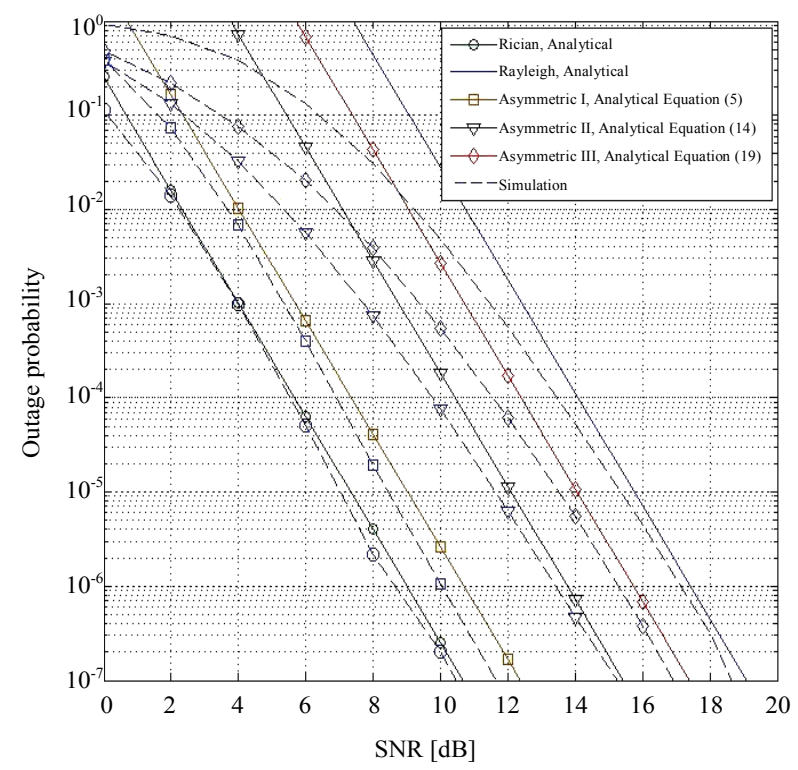

Figure 2. The outage probability over asymmetric channel I, asymmetric channel II and asymmetric channel III. Due to the high SNR approximation for the analysis, analytical results converge with Monte-Carlo simulation results at medium and high SNR regime. scenario II highly improves the outage performance. It is clear from the above discussion that S-R is a dominating link, therefore, it is better to localize the opportunistic relay node in LOS environment with respect to $\mathrm{S}$ in order to improve the overall outage performance. Finally, the Monte-Carlo simulation results provided in Figure 2 shows that the analytical outage probabilities are a tight bound at medium and high SNR regime.

\section{Conclusions}

In this letter, the outage performance of opportunistic AF relaying over asymmetric and i.n.d fading environments has been investigated. A lower bound of the outage probability has been derived and validated through Monte-Carlo simulation results. We show that the outage performance is better when the relay is in LOS situation with respect to the source rather than to the destination.

\section{Acknowledgements}

The authors would like to thank the European IST-FP7 WHERE project and the European Network of Excellence NEWCOM++ for support of this work.

\section{References}

[1] J. N. Laneman, D. N. C. Tse and G. W. Wornell, "Cooperative Diversity in Wireless Networks: Efficient Protocols and Outage Behavior," IEEE Transactions of Information Theory, Vol. 50, No. 12, 2004, pp. 3062-3080.

[2] Y. Zhao, R. Adve and T. J. Lim, "Outage Probability at Arbitrary SNR with Cooperative Diversity," IEEE Communications Letters, Vol. 9, No. 8, 2005, pp. 700-703.

[3] Y. Zhao, R. Adve and T. J. Lim, "Symbol Error Rate of Selection Amplify-and-For- ward Relay Systems," IEEE Communications Letters, Vol. 10, No. 11, 2006, pp. 757759.

[4] A. Bletsas, H. Shin and M. Z. Win, "Cooperative Communication with Outage Optimal Opportunistic Relaying," IEEE Transactions on Wireless Communications, Vol. 6, No. 9, 2007, pp. 3450-3459.

[5] M. Katz and S. Shamai, "Relaying Protocols for two Colocated Users," IEEE Transactions on Information Theory, Vol. 52, No. 6, 2009, pp. 2329-2344.

[6] H. Suraweera, G. Karagiannidis and P. Smith, "Performance Analysis of the Dual-Hop Asymmetric Fading Channel," IEEE Transactions on Wireless Communications Letters, Vol. 8, No. 6, 2009, pp. 2783-2788.

[7] K.-S. Hwang, Y.-C. Ko and M.-S. Alouini, "Performance Analysis of Incremental Opportunistic Relaying over Identically and Non-Identically Distributed Cooperative Paths," IEEE Transactions on Wireless Communications, Vol. 8, No. 4, April 2009, pp. 1953-1961. 